\title{
Improving Accessibility to Intangible Cultural Heritage Preservation using Virtual Reality
}

\author{
ELMEDIN SELMANOVIĆ, University of Sarajevo, Faculty of Natural Sciences and Mathematics \\ SELMA RIZVIC, University of Sarajevo, Faculty of Electrical Engineering \\ CARLO HARVEY, Birmingham City University, Faculty of Computing and the Built Environment, \\ DUSANKA BOSKOVIC, University of Sarajevo, Faculty of Electrical Engineering \\ VEDAD HULUSIC, Bournemouth University, Faculty of Science and Technology, \\ MALEK CHAHIN, University of Sarajevo, Faculty of Natural Sciences and Mathematics \\ SANDA SLJIVO, University of Sarajevo, Faculty of Electrical Engineering
}

\begin{abstract}
Presentations of virtual Cultural Heritage artifacts are often communicated via the medium of interactive digital storytelling. The synergy of a storied narrative embedded within a 3D virtual reconstruction context have high consumer appeal and edutainment value. We investigate if $360^{\circ}$ videos presented through virtual reality further contribute to user immersion for the application of preserving Intangible Cultural Heritage. A case study then analyses whether conventional desktop media is significantly different than virtual reality as a medium for immersion in intangible heritage contexts. The case study describes bridge diving at Stari Most, the old bridge in Mostar Bosnia. This application aims to present and preserve the bridge diving tradition at this site. The project describes the site and history along with cultural connections, a series of quiz questions are presented after viewing all of the materials. Successful completion of the quiz allows a user to participate in a virtual bridge dive. The subjective evaluation provided evidence to suggest that our method is successful in preserving intangible heritage and communicating ideas in key areas of concern for this heritage that can be used to develop a preservation framework in the future. It was also possible to conclude that experience within the VR framework did not affect Effort Expectancy for the web application, but the same experience made a significant influence for the Performance Expectancy construct.
\end{abstract}

CCS Concepts: • Human-centered computing $\rightarrow$ HCI design and evaluation methods; $\bullet$ Computing methodologies $\rightarrow$ Virtual reality; • Applied computing $\rightarrow$ Education.

Additional Key Words and Phrases: virtual reality, intangible cultural heritage, head mounted display, storytelling

ACM Reference Format:

Elmedin Selmanović, Selma Rizvic, Carlo Harvey, Dusanka Boskovic, Vedad Hulusic, Malek Chahin, and Sanda Sljivo. 2019. Improving Accessibility to Intangible Cultural Heritage Preservation using Virtual Reality. ACM f. Comput. Cult. Herit. 1, 1, Article 1 (January 2019), 20 pages. https://doi.org/10.1145/3377143

Authors' addresses: Elmedin Selmanović, eselmanovic@pmf.unsa.ba, University of Sarajevo, Faculty of Natural Sciences and Mathematics, Selma Rizvic, srizvic@etf.unsa.ba, University of Sarajevo, Faculty of Electrical Engineering, Carlo Harvey, carlo.harvey@bcu.ac.uk, Birmingham City University, Faculty of Computing and the Built Environment, Dusanka Boskovic, dboskovic@etf.unsa.ba, University of Sarajevo, Faculty of Electrical Engineering, Vedad Hulusic, vhulusic@bournemouth.ac.uk, Bournemouth University, Faculty of Science and Technology, Malek Chahin, mchahin@pmf.unsa.ba, University of Sarajevo, Faculty of Natural Sciences and Mathematics, Sanda Sljivo, sanda.sljivo@etf.unsa.ba, University of Sarajevo, Faculty of Electrical Engineering,

Permission to make digital or hard copies of all or part of this work for personal or classroom use is granted without fee provided that copies are not made or distributed for profit or commercial advantage and that copies bear this notice and the full citation on the first page. Copyrights for components of this work owned by others than ACM must be honored. Abstracting with credit is permitted. To copy otherwise, or republish, to post on servers or to redistribute to lists, requires prior specific permission and/or a fee. Request permissions from permissions@acm.org.

(c) 2019 Association for Computing Machinery.

XXXX-XXXX/2019/1-ART1 \$15.00

https://doi.org/10.1145/3377143

ACM J. Comput. Cult. Herit., Vol. 1, No. 1, Article 1. Publication date: January 2019. 


\section{INTRODUCTION}

Cultural Heritage can be popularly divided into two categories: tangible culture and intangible culture [65]. The focus of tangible heritage is on buildings, monuments, artifacts, art works, and other cultural property. The term Cultural Heritage has traditionally been used to refer to tangible heritage. However, the meaning of the term has been extended to include intangible heritage in the last decades of the 20th century. UNESCO's defined intangible heritage includes [64]:

- "oral traditions and expressions, including language as a vehicle of the Intangible Cultural Heritage;

- performing arts;

- social practices, rituals and festive events;

- knowledge and practices concerning nature and the universe;

- traditional craftsmanship."

Traditional methods aimed at digitizing intangible heritage are currently using basic storage media such as audio and video recordings, which are good tools for documenting. More recently, techniques for documenting intangible heritage have begun to explore more contemporary technologies [20,58]. The aim of these efforts was to preserve Intangible Cultural Heritage and to allow its use in an educational context. This could be communicating dance choreography or the immersion of a particular environment, but in the end it is better described as documenting the intangible heritage and there is a difference between documentation and preservation [40]. For intangible heritage and preservation, it is important to convey context and the experience of the heritage community. In this work, we explore the use of digital storytelling techniques to preserve the context of the intangible heritage through a case study and use Virtual Reality (VR) to provide immersion, presence and experience.

Virtual environments (VEs) can provide a means by which Intangible Cultural Heritage sites can be rigorously explored and investigated. This is a function of many elements, the most important of which is probably a Cultural Heritage site reconstruction. Realism can be misleading because it implies a degree of certainty about the environment [30]. Due to the interpretation it provides through participant inference, it is important to address the uncertainty surrounding a VE. This is to preserve the Cultural Heritage sites governing historical context. Some standardised methods for digitally preserving the past have been developed [1, 7, 19, 26]. Unfortunately, the following key issues relating to intangible heritage are not actively addressed in this area of work [63]:

(1) Validity: historical records may result in subjectivity and misinterpretation of many intangible heritage functions;

(2) Inclusivity: the expressions of Intangible Cultural Heritage are those shared by other cultures. These developments may be from common roots or alternatively from distinct roots;

(3) Representativity: this factor is mitigated by scientific approaches of the modern era, e.g. we can record dances or measure materials. Recreating the past, however, requires intelligent data extrapolation, yet the need to be representative of knowledge and tradition, skills and customs is still required;

(4) Temporality: an entirely different precept is to experience something through the lens of a different culture, even ignoring the implications of hardware and software that are not non-invasive. Intangible heritage not only represents heritage, but also reflects contemporary heritage;

(5) Sensitivity: current religious and political themes may affect the agenda of recreating intangible heritage, traditional or contemporary;

(6) Community: intangible heritage is such only when the communities that create, maintain and transmit it are recognised.

Assessing a reconstruction of Cultural Heritage is not necessarily deterministic. It can use quantitative or qualitative processes or a mixed methods approach [45]. Tangible heritage can be assessed quite readily. The availability of commodity hardware and techniques such as spectroradiometers, laser scanning, ground penetrating 
radar and reflectance transformation imaging enables numerical quantities to be evaluated goniometrically, radiometrically and perceptually. Intangible heritage, however, is more difficult to evaluate. The reality is that validation will take the form of an opinion if evaluation is required. This even applies to quantitative information bearing inherent uncertainty due to extrapolative processes [11]. Validation should occur considering the quantitative and qualitative. The validation should come from contemporary practitioners in the context of intangible heritage and considering the Community element of the concerns.

This work's main contributions are:

- An investigation of Performance Expectancy and Effort Expectancy across VR and Web Multimedia representations of technological approaches to preserve intangible heritage;

- Suggest up-to-date methods for the preservation of Intangible Cultural Heritage. This is facilitated through a case study to preserve the Mostar bridge diving tradition via VR and Web-based media;

- The user experience evaluation is designed and conducted to identify predictive and explanatory use measures for the technologies;

- A comparison of VR and Web Multimedia approaches to preservation of intangible heritage is presented and analysis is conducted around user perception exposed to the web-condition with no prior exposure to VR, and with prior exposure.

It should be noted that this contribution is an extension of the work by Selmanović et al. [56].

\section{RELATED WORK}

A variety of techniques inform the paradigm of creating a VR or Web-based experience for Intangible Cultural Heritage. This section reviews prior work on Interactive Digital Storytelling Systems and investigates what we can learn from these processes. We follow this up with a discussion of Interface design to support design choices taken in the development of the application. Finally we look into evaluation methodologies with specific focus on Technology Adoption protocols as this is a study into a disruptive technology for a specific new purpose: Intangible Cultural Heritage.

\subsection{Interactive Digital Storytelling Systems}

Athena Plus [47] makes recommendations for cultural institutions that strongly encourage the transmission of information about Cultural Heritage through digital storytelling. For Interactive Digital Storytelling (IDS) systems according to [55], there are many challenges in designing and developing story worlds. Among them is the Narrative Paradox challenge [8]. This challenge is defined by Henrik Schoenau-Fog as a conflict between the freedom of choice of a user and the control of the main storyline [55]. When stories are linked to objects in these environments, interactive virtual environments as parts of IDS encounter this problem. Users might miss finding these triggers and not perceiving important information as a result. Thus, narrative paradox solutions are significant contributions to an IDS methodology.

There are several works arguing in favor of emerging narratives as possible solutions to this challenge $[38,51,61]$. These are presented in the form of stories that emerge from the players' interaction with the gameplay governing systems. This proposed solution could not be taken into consideration as our case study has already predefined stories.

As Champion states in [12], although our approach has game elements, it cannot be considered as traditional Serious Games as authors have described in [4].

Argyriou et al. [6] propose a conceptual gamification framework for VR applications based upon the use of game elements in a $360^{\circ}$ video environment to enhance user interaction with a case study of the Cultural Heritage site, in Rethymno city, Greece. The work presented by Argyriou et al. introduces a similar case study to the 
work presented in this paper. It uses $360^{\circ}$ video to convey information, it has a quiz with questions from videos, and it has a motivational factor. The main advantage of these approaches is that the game presented has good replayability value, which is not the case with our case study.

Ivkovic et al. [33] also use $360^{\circ}$ videos for representation of Cultural Heritage in presenting the Bridges of Sarajevo. The user study that is presented with this work has shown that users like $360^{\circ}$ videos and the freedom in choosing the order of the stories, in addition to the reward at the end. The advantage is a high level of immersion and the user study corroborates this as it is reported that a significant number of users felt like they were walking on the real bridge while watching the $360^{\circ}$ videos.

A cinematic use of $360^{\circ}$ videos and their application in Cultural Heritage is also described by Dowling et al. in [21]. The authors made a $360^{\circ}$ video about the Faoladh legend in late 9th century Ireland and uploaded it on YouTube. Afterward, they analysed YouTube Analytics of that video.

Elmezeny et al. in [22] also consider $360^{\circ}$ videos and their two dimensions of immersion: technical and narrative. Like in our case, they did a user evaluation with a comparison between $360^{\circ}$ videos and traditional ones.

Russia's Hermitage Museum [44], in partnership with the Russian video production company Videofabrika, has created a novel VR experience for visitors, called The Hermitage VR Experience. This takes the form of a 19-minute movie in $360^{\circ}$ format. This is a new format in historical pedagogy through an interactive and immersive experience.

When considering historical pedagogy, it is essential to mention a paper by Sylaiou et al. [60] where authors measure the educational impact of diverse technologies in online virtual museums.

Christopoulos et al. [13] present another project that uses IDS and VR to represent Intangible Cultural Heritage. The authors describe the Battle of Thermopylae as an interactive VR application. The application consists of a 3D format and IDS game introductory video. What is interesting and different from our project is that for Head Mounted Displays (HMDs), the authors chose a Powerwall type of VR. They chose this because they wanted to make the application accessible to large groups of people visiting the museum as well as taking into account the social aspect of a visit to the museum.

Ashima et al. [62] uses a combination of emotional storytelling, VR and game elements to awaken people's awareness of the dangers of plastic. This approach is like ours in a way that it wants to get an emotional reaction from users, and it uses similar methods to accomplish this.

2.2 Interface

Virtual reality provides a computer-generated environment experience, typically delivered through a VR headset as an audio-visual stimulation. These indications simulate physical presence in a VE with a potential addition of olfactory, tactile, and other modal integrations. The interaction is one of the key aspects related to immersion, a system's ability to produce a sense of presence. Interaction has been investigated in many areas, from interactive multimedia instructional [57] and interaction between human and computer [59]. The interaction mode can be divided into several parts: locomotion - to navigate through the virtual environment; selection - scenariodependent mode, used to interact with virtual objects; manipulation - to change the position, orientation or shape of an object; scaling - to explore object details (scaling up) or environment (scaling down); and menu interaction - to perform activity. Our study focuses on the selection and menu interaction that is directly correlated and should therefore be considered holistically.

Two elements are essential for selection: identification, so that the systems know which object to select; and a command to select the identified object. The selection may be local, where the object is within reach, and at a distance where the user is unable to reach the desired object. Through laser beams, spotlights, gaze selection or voice input, the latter can be achieved. Mendes et al. [42] proposed a selection taxonomy that includes cardinality - the number of objects that can be selected simultaneously and gradual refinement-gradual subselection among 
multiple larger groups of initially selected objects. Argelaguet and Andujar [5] propose a comprehensive survey with a classification of selection techniques.

Menu interaction is a vital aspect of many interactive systems, enabling additional, extended functionality to be added. While in conventional 2D interfaces this is a very common interaction style, it is an actively researched topic in the 3D VE domain. Different techniques have been explored since the emergence of VR applications and taxonomies have been proposed [15]. One approach is to use common 2D menu types in 3D VR environments, such as pop-up and pull-down menus [35], which can be fixed to the view port of the camera, used as a heads-up display (HUD), or floating in 3D space, with predefined position and orientation $[10,14]$. The other approach is to use more natural paradigms in 3D space with "physical" 3D menus, e.g. spin / ring menus [25]. Finally, those using diegetic interfaces are the most natural menu implementation in 3D VEs [52]. As part of the game world, these interfaces exist and are visible to the player and the player's character. However, selecting a suitable type of VR menu should be based on interaction style and purpose, application domain, and possibly other factors. See $[15,37]$ for a comprehensive overview of 3D menu paradigms and taxonomies.

\subsection{Evaluation}

Researchers frequently use the Technology Acceptance Model (TAM) proposed by [16] to evaluate user attitude and behavioral intent towards the use of a new technology. Reasonable Action Theory was adapted and referred to as the Technology Acceptance Model (TAM) by [17]. The TAM is used to model the acceptance of technology by users and their use at work. Two constructs explain user motivation in the basic version of TAM: perceived usefulness (PU) and perceived ease of use (PEU).

Perceived ease of use refers to the degree to which the user expects effortless use of new technology. Not only are external factors influencing these two constructs, but authors show that PEU also influences PU. The attitude of users towards the use of new technology is affected by both PU and PEU, and this leads to potential actual use of the system. Later, a modified version of TAM was developed by [18]. A new factor was introduced in this version apart from PU, PEU, and actual usage: intention to use. The intention to use influences both PU and attitude towards use, which in turn affects the actual use of the new technology.

Venkatesh and Davis defined the latest version of TAM [66]. They found out that both PU and PEU have a direct influence on behavior intention, so they replaced the attitude towards use and intention to use with behavioral intention factor which influences the use behavior. Ultimately, based on previous research, the Unified Theory of Acceptance and Use of Technology (UTAUT) was developed by Venkatesh et al. [67]. UTAUT has four predictors of the behavior of the user: PU involved in Performance Expectancy, PEU in a construct called Effort Expectancy, and new predictors of the behavior of the user: social influence and facilitating conditions. Figure 1 shows the UTAUT block diagram.

VR's recent popularity presents researchers with the challenge of finding relevant determinants of VR acceptance as well as the advantages of using VR compared to other digital content technologies. Bertrand and Bouchard (2008) [9] have tested how the Technology Acceptance Model (TAM) applies to the use of virtual reality in clinical settings and have shown that intention to use VR is only predicted by Perceived Use. Fagan et al. (2012) [24] explored the combined influence of personal innovation in the IT domain and the basic constructs of TAM: perceived ease of use and perceived usefulness. Their case study involved the use of a medical emergency crash cart by nursing students and VR simulation. The authors found that intent to use was explained by the TAM constructs and also showed that the perceived ease of use had a significant positive relationship with perceived usefulness. Manis and Choi (2018) [39] explored the extension of the (TAM) by incorporating perceived enjoyment into the acceptance of VR hardware as a key belief variable. 


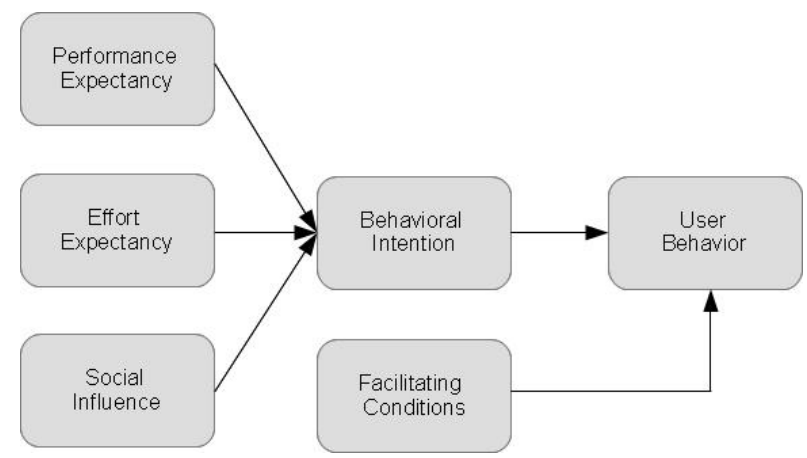

Fig. 1. Unified Theory of Acceptance and Use of Technology - UTAUT, adopted from [67]

\section{MOSTAR BRIDGE DIVING}

The diving takes place in Mostar, a town in Bosnia and Herzegovina famous for its Old Bridge. It is an Ottoman bridge, designed by Mimar Hayruddin, a student and apprentice of the famous architect Mimar Sinan and built over the Neretva River in the 16th century. During the aggression on Bosnia and Herzegovina, the bridge was destroyed by Croatian military forces on 9th November 1993 and rebuilt in 2004. Diving from this bridge is a tradition that began in 1664 and is today preserved as part of the Intangible Cultural Heritage of B\&H. The aim of the project is to present through VR storytelling and virtual diving the history of the Bridge and cliff diving tradition. Users watch short VR stories about the Bridge, its history, destruction and reconstruction, and they can make a virtual jump through a VR computer simulation if they evidence, by means of a quiz, enough learning from the VR storytelling.

\subsection{VR Storytelling}

The project structure is shown in Figure 2. Users select stories from the 3D menu after an intro story. They perform a quiz after watching all the stories to assess how much they have learned. If the questions from stories are answered correctly, they can proceed to the simulation of the virtual bridge dive. Everything is in $360^{\circ}$ since the application is designed for a Head Mounted Display: the stories, the user interface, the quiz and the simulation of bridge diving. The link to download the executable application of the project is provided online [54].

Digital storytelling follows the rules of storytelling typically used in other media like the theatre and film. Within the Sarajevo Charter platform [28], we defined the guidelines for interactive digital storytelling and most of these guidelines have been implemented in the stories for this project. VR video, however, brings new challenges with it, as those rules no longer apply. For example, the story as defined in film language grammar is not staged or directed as our viewer can now turn around and watch the content in a $360^{\circ}$ field of view. VR stories need to convey the information to the user while using the VEs immersive potential.

The storytelling has been structured into five stories for this project. The story themes are Introduction, History of the Old Bridge, Destruction and Reconstruction, Architecture of the Old Bridge and the Interview with a Contemporary Diver. This structure is shown in Figure 2. The introduction story provides an historical overview of the Old Bridge, the bridge diving tradition and the content of the application. It is a combination of the voice-over narration and Bridge's $360^{\circ}$ videos. The stories about the Bridge's history, its architecture, destruction, and reconstruction are similarly implemented with $360^{\circ}$ videos recorded in and around the Bridge in different positions. The story of bridge diving takes place as an interview with Lorens Listo, the famous champion 


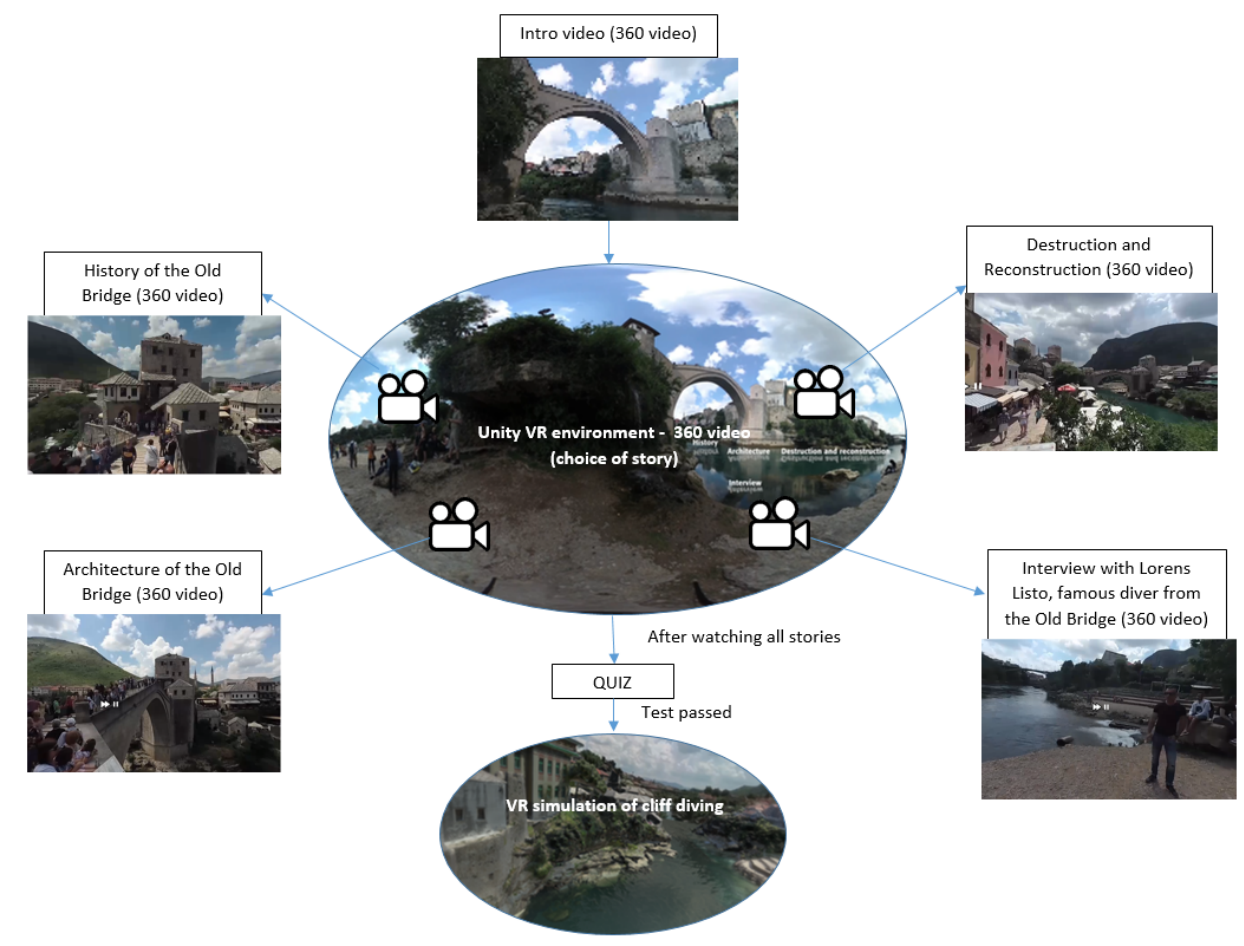

Fig. 2. The structure of the Mostar bridge diving application

of bridge diving, and a city legend. He describes the tradition of bridge diving, competitions, the types of jumps performed in competition, as well as his emotions for this sport and motivation to devote his life to it.

A Garmin VIRB 360 Action Camera recorded all VR videos. Two microphones (Sennheiser ew 100 ENG G3 Wireless Microphone Combo System) and one audio recording device (Tascam DR40) recorded the sound for the interview separately. We chose not to spatialize the sound and chose to present sounds in a binaural fashion. This interferes with the user immersion [32], however, and this is confirmed in the user experience evaluation results. The guidelines for interactive digital storytelling [50] are followed as much as possible. We did not use actors in this case, but the stories still have a unique visual identity. Professionals were involved in all fields of content creation, the content was divided into sub-stories that can be viewed independently, and the stories are short, dynamic and informative. The motivation factor was introduced, as emphasised in [50] and [49], to inspire users to watch all the stories and solve the narrative paradox. In this project, the motivation is to experience bridge diving through the VR simulation. This is enabled if the user passes the quiz to the questions framed around the video story information with sufficient correct answers (60\% threshold).

We created a WebGL application with the same content for users who do not have access to Head Mounted Displays [27]. Navigation and interaction with VR videos and environments through mouse and keyboard commands are implemented in this application. We will compare two experiences in the Evaluation section to show the advantages of implementing VR through HMDs over the conventional Web version. 

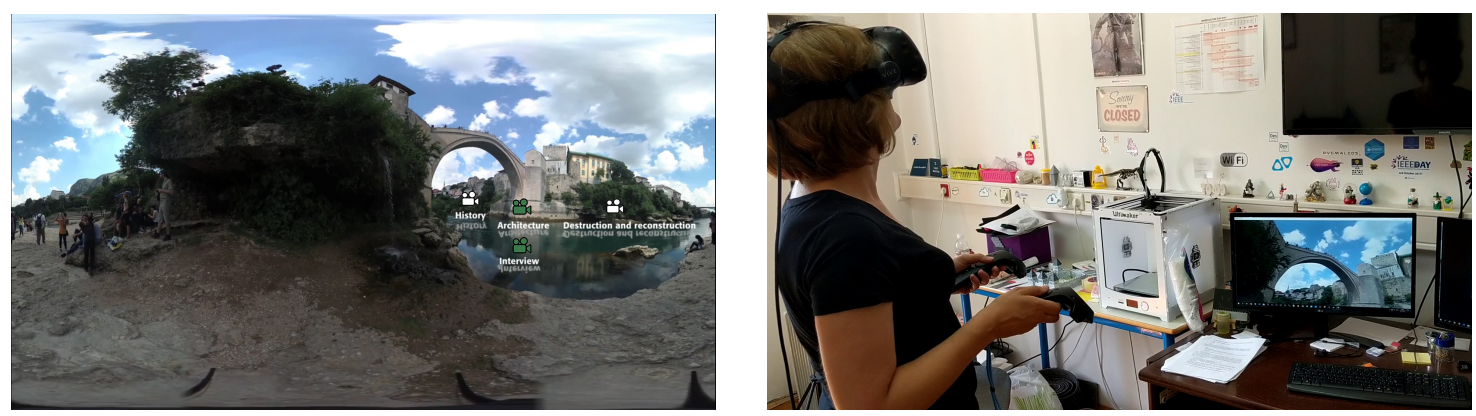

Fig. 3. Panorama of the main menu: the story selection - the green camera button indicates the story has been watched (left). User during the evaluation experiment (right). A full video highlighting key elements of this experimental process is available online [53].

\subsection{User Interface and Navigation}

Both local and at-a-distance interactions were used in our VR system. The former was used in the diving simulation to climb the ladders, while the latter was used in the quiz to navigate the digital stories and provide answers. Furthermore, in the diving simulation, natural interaction was used for locomotion.

The introduction story will be played automatically once the application is started. Upon completion, the user will be taken to the main menu where one of the four stories could be selected. The menu was implemented using Unity Canvas UI and Button UI, the latter consisting of the camera icon and the label, Figure 3 . The buttons have been integrated into the VE so that it appears as if floating in the river, with the reflection of the label on the surface of the water. There are two states in the camera icon: not watched - appears white, and watched - appears green. Once opened, the user can watch the selected story and either pause it or skip it, using the floating 2D menu available in all stories, Figure 4. A new menu item, labeled "Take the Quiz" appears above the river, after all four stories have been opened at least once.

The quiz scene is a room with a few windows, a door and some traditional Bosnian objects: a Bosnian rug and "Peškun" - a traditional six, eight or nine-sided carved table or chair, Figure 5. The user could see the Old Bridge through the windows, giving the impression that he or she is right next to the bridge in one of the towers.
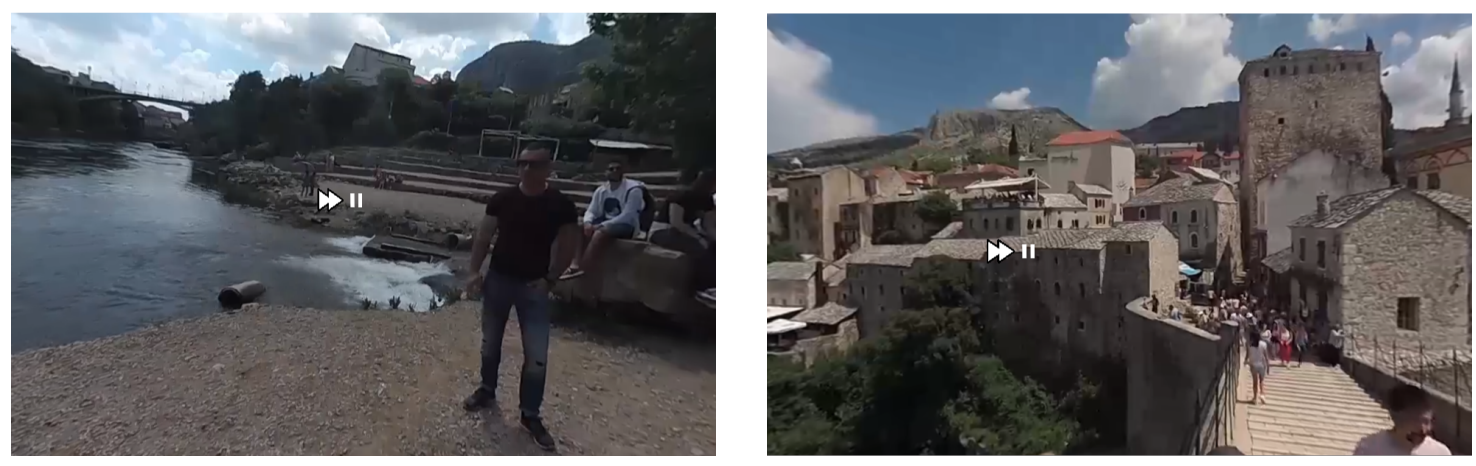

Fig. 4. Story controls in "Interview" story (left) and "History" story (right) - left button for skipping the current story and moving to the main menu, and right button to play/pause the story. 

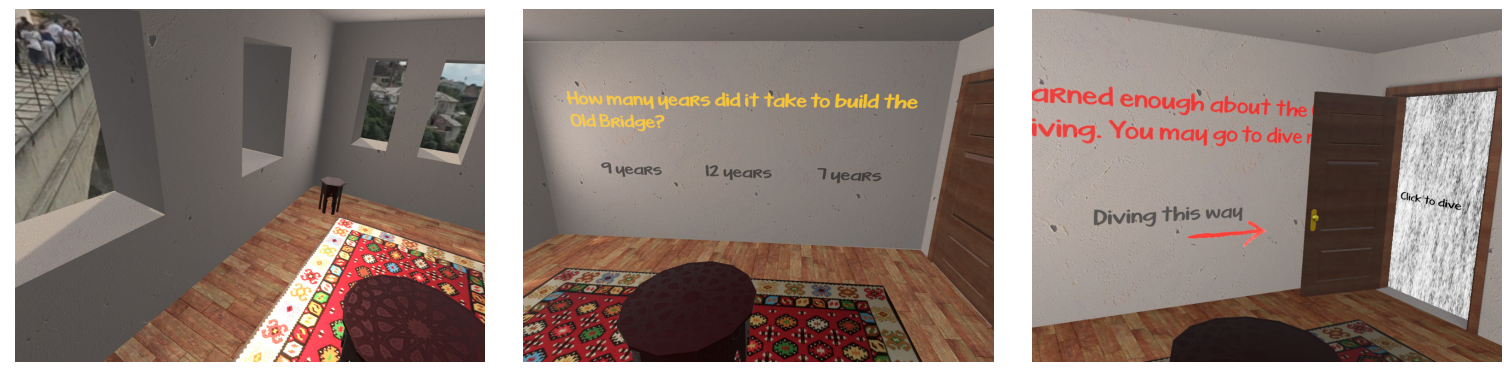

Fig. 5. Screenshots of the room where the quiz was taking place. The user could see some traditional Bosnian furniture in the room and the Old Bridge through the windows (left). The first question (middle) - each question was displayed on the left, front or right wall. The direction for progression to the diving component once the quiz participation was successful (right).
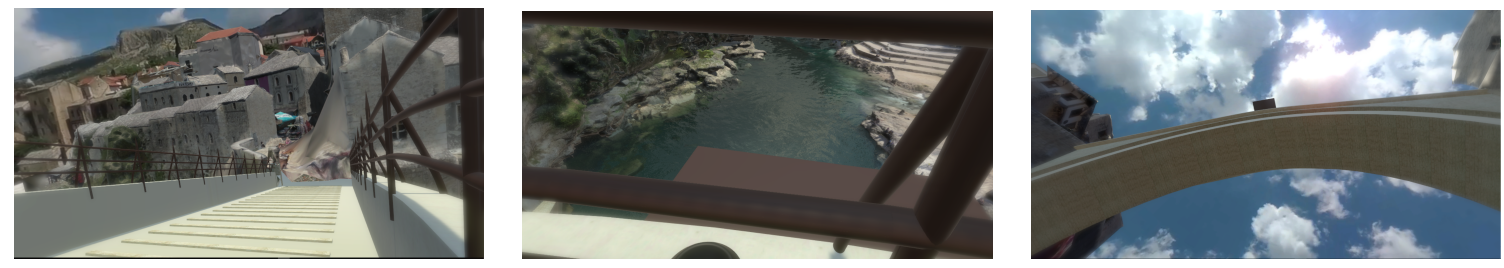

Fig. 6. Screenshots from the simulation of the Old Bridge dive. The user starts the simulation at the start of the Old Bridge (left). View of the river from the top of the diving platform (middle). The view looking back up at the bridge after completing a successful dive from the Old Bridge in VR (right).

The quiz interface has been made diegetic, mixing the quiz questions and multiple-choice answers with the 3D environment, so it looks like they have been painted on the walls, Figure 5 (middle). Question appearance is controlled by scripts. A virtual laser pointer is used, as in the main menu, to interact with the quiz menu and to answer the questions. As the user progresses, the score is calculated and kept in the background.

3.2.1 Quiz Feedback. Several changes have been made to the quiz based on the internal discussion between the authors and the comments received in the initial user evaluation. In the initial implementation, within one of the three canvases, there were five hard coded questions in the quiz, each shown on request in its panel. For several reasons, this implementation was sub-optimal: users always had the same questions to answer, both questions and answers always appeared in the same order, there were as many panels as there were questions, adding new questions would require the project and scripts to be re-implemented, etc.

Without UI panels, the new implementation is fully dynamic. For the questions, each of the three canvases, placed on the room's three walls, has one text and three UI button placeholders and offers answers respectively. Their text fields are stored in a text file from a question pool. Text controllers randomize their presentation order. A question controller reads and displays the first question, from a random order, on the appropriate wall. The controller then displays the other $n-1$ questions [ $n$ is the total number of questions in the quiz] one by one on a random screen, i.e. a room wall. The arrow indicator and the message "Turn [direction] to a new question" where direction is set to either left or right is displayed on the wall of the question answered. The system checks if the user answered more than $60 \%$ of the questions correctly when answering the last question. If so, a confirmation message is displayed and a link to the diving simulation will direct the user to the opening door. Otherwise, the message "You had too many incorrect answers. Please go back and watch the stories again." and the link to the stories is shown to allow the user to listen to the stories and take the quiz again, Figure 7 (left). 

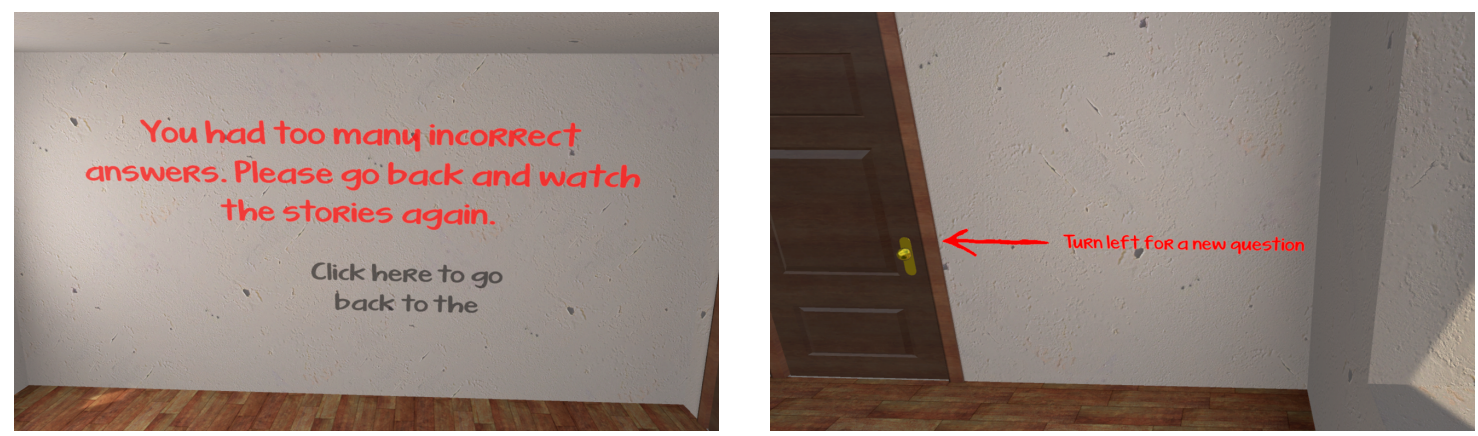

Fig. 7. Messages in the quiz: instruction to watch the stories again when answering incorrectly to more than $40 \%$ of the questions (left), and the instruction to turn left for a new question (right).

The second improvement was related to the user feedback obtained in the first study. Several users reported that the next question indicator was too ambiguous and some of them tried clicking on it, instead of turning towards the indicated direction. The reasons could be found either in the instruction or the colours used, or both of them. In the current implementation, the instruction has been changed to clearly guide the user to turn towards the new question. In addition, both the text and the arrow are displayed with a fade-in effect and in red colour, making it different from the [yellow] interactive elements, Figure 7 (right).

\subsection{Simulation}

The simulation is implemented in Unity game engine. Virtual Reality Toolkit (VRTK) [68], helped solve problems with locomotion. The user's movement is handled by means of arm-swinger system technology, where the user moves by pressing a button on each controller and swinging arms. Using VRTK's grab attachment mechanics system, the feature of climbing a diving platform is implemented. This simulation uses an Old Bridge in Mostar polygonal model that is accurately created on a real-world scale. Blender and SketchUp tools were used to model and texture.

The simulation allows the user to actually dive into and come out of the Neretva river to improve immersion. Once the player enters the water, a force is put into effect on the player object. This affects their velocity when entering the water by a controllable value relative to the normal water surface: $p_{v}=p_{v}+s \hat{N}$, where $p_{v}$ is the vector component of the player's velocity, $s$ is a scalar and $\hat{N}$ is the normal unit vector of the water surface. This is evaluated until the water surface is reached by the player. Once the player reaches the surface, after several seconds of movement and possible swimming with the arm-swinger technology, the simulation takes the player back to the beginning of the dive simulation and allows the user to replay if desired, allowing this component of the VR application to be replayed.

3.3.1 Drone-based Photogrammetry. One of the comments from the initial user evaluation, which was addressed in the extended and improved version of the project had been lack of the Old Bridge surrounding area. This area is considerable in size and would be time-consuming to reconstruct manually. Therefore, it was decided to use photogrammetry for reconstruction as it was already proven to be feasible for Cultural Heritage site reconstructions in previous research $[3,29,41,43,46,48,69,70]$.

Terrain configuration around the Old Bridge was such that it did not allow access by foot. It was necessary to capture photographs which would provide accurate photogrammetry results. Therefore, the DJI Mavic 2 Pro drone was used to capture footage of the area. Both sides of the Neretva river south of the bridge (side from which dives are made) were video recorded in $4 \mathrm{~K}$ resolution at 30 frames per second. Images were captured in a 

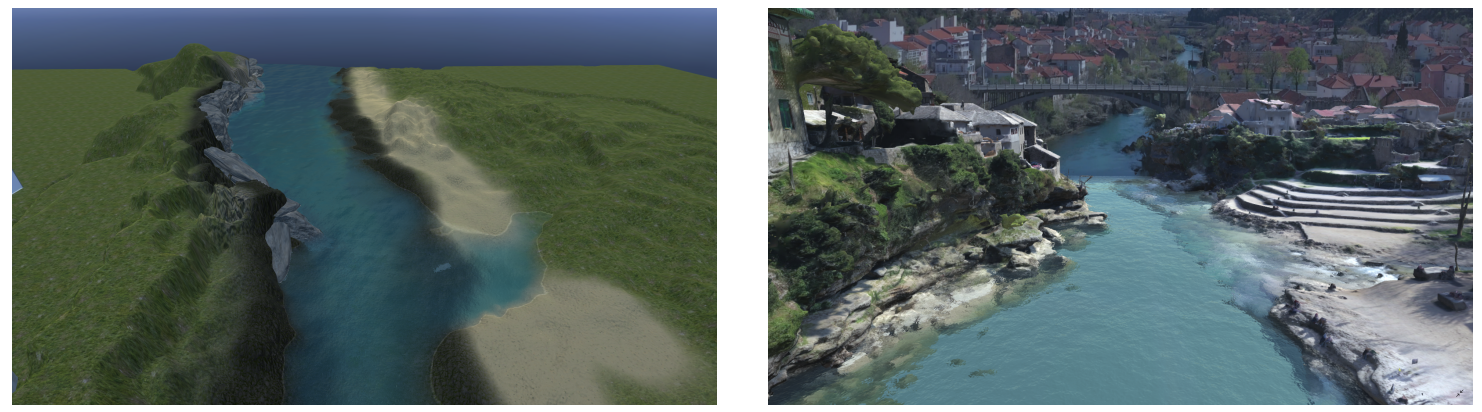

Fig. 8. Old Bridge surroundings: limited surroundings in the initial study (left), and photogrammetry reconstructed surroundings (right).

horizontal scan pattern from 5 different height levels. Video recording was used to extract around 600 frames to be used as input into the photogrammetry software.

For the reconstruction of 3D surrounding, commercial photogrammetry solution RealityCapture was used. It required around 30 hours to process the frames, on a PC with an i7 processor, 16 GB RAM, and dedicated GPU with 4 GB of memory. Software generated a point cloud, 3D geometry and textures. The model initially consisted of 40 million faces, but was simplified and retopologised to half a million faces to improve performance of the application.

Corrections to the geometry and textures were performed in Autodesk Maya and Adobe Photoshop. Buildings which had rough surfaces were smoothed, extra geometry not visible in the simulation was removed, and the quality of the textures and their mapping for the near buildings was improved, results of this process can be seen in Figure 8. Finally, the model of the Old Bridge surrounding was imported into Unity.

\subsection{Web-based Version}

In order to provide a comparison with the VR version, a web-based version needed to be created. The VR version had a number of features that provided interaction with VR-specific interfaces such as the virtual laser pointer for button interaction, controller support to climb the ladder for the dive and $360^{\circ}$ display interaction. These were not supported when naturally compiling for a WebGL version. Additionally, the project is quite large so a number of optimisations to reduce loading times for the client were made. These included stripping engine code and texture downsizing. For the interaction support previously mentioned, click-through in screen space was added to support the replacement of the virtual laser pointer, climbing of the ladder is deterministic on approach and ascent now rather than via VR interaction with the controllers and finally standard WASD keyboard movement and mouse look controls were integrated to replace the more natural interaction afforded through VR.

\section{EVALUATION OF VR IMPORTANCE}

The study objective was to evaluate the importance of VR technology for user experience of the Mostar Old Bridge diving application. The importance of VR technology is measured indirectly by measuring user experience of the web version of the same application, comparing opinions of two groups:

- users with prior experience of VR Mostar diving application (Web after VR group);

- users without prior experience of VR Mostar diving application (Web Only group).

Our study focused on assessing user inclination and likelihood to use the Mostar bridge diving web application [27] by measuring the main predictors: ease-of-use and usefulness. We have selected to use a unified model for 
technology acceptance - UTAUT [67], with the following basic constructs related to ease-of-use and usefulness respectively: Effort Expectancy and Performance Expectancy.

The results for the two groups will be analyzed and compared. Further on we will explore user Behavioral Intention dependent on the technology type, VR or web-based. Based on the review of related research, and our experience with the pilot evaluation of user experience, the following hypotheses were made:

H1: VR experience has a positive effect on the web application Performance Expectancy;

H2: VR experience has a negative effect on the web application Effort Experience;

H3: VR experience will have a significant positive influence on Behavioral Intention to use the VR.

\subsection{Methodology}

We adopted a quantitative approach based on a user survey as the preferred method for technology acceptance and user behavior research. Web based survey questionnaires were designed and implemented to measure the identified constructs of importance.

Since our study is based on previously confirmed theoretical models we designed the questionnaires analogous to these described instruments $[2,9,17,18,39,67]$. Usually authors modify the question items to suit the specific study and the designed instrument has to be tested to establish its validity and reliability [2]. Items representing Performance Expectancy are highly linked to the specific usage of the application, and quite different if the application is used in a business context.

We have designed two questionnaires. The first questionnaire was designed to measure Performance Expectancy and Effort Expectancy of the web application, and was distributed to both groups, Web After VR and Web Only.

The second questionnaire contained items comparing the VR and web application over the PE and EE constructs but also including a new construct aligned to Behavioural Intention. This questionnaire was distributed only to users who tried both applications, i.e. the Web After VR group.

The first questionnaire contained seven-point Likert items for measuring the identified constructs, using 8 items for the Performance Expectancy and 5 items for Effort Expectancy. The questionnaires were peer reviewed by psychometric and UX experts. Prior to validating our hypotheses we tested the validity and reliability of the instrument.

The second questionnaire, which was used for direct comparison of VR and the web application, was divided in sub-scales linked to the same constructs. This contained seven-point Likert items, with 1 indicating strong preference of the web application, 4 indicating equality, and 7 indicating VR application preference. The comparison of constructs were measured using 5 items for the Performance Expectancy, 6 items for the Effort Expectancy, and 2 items for the Behavioral Intention. Within the usefulness sub-scale (Performance Expectancy) we repeated items from the first questionnaire focused on immersion, and within the ease-of-use sub-scale (Effort Expectancy) we have repeated all items and add an item to compare overall ease-of-use (EE.6 in Figure 9).

\subsection{Evaluation Experiment}

The experiment involving usage of the VR application was conducted at the Graphics Lab at the University of Sarajevo. Engagement with the web application did not require user presence at the lab.

Participants were recruited by invitation, users were invited so as to represent different groups including students and professionals, representatives of different user types with respect to their professional background and gender. In order to ensure that the responses represent a diverse, cross-section of respondents and to establish the validity of the study we asked participants to provide relevant demographic data: age, gender, study/work profile [36].

The Web after VR group included 52 participants. Users were invited to participate in the interactive digital stories and dive from Mostar Old Bridge's virtual model, shown in Figure 3. Individual evaluation sessions were 


\begin{tabular}{|c|c|c|c|}
\hline Questions & Responses & Web after VR & Web Only \\
\hline \multirow{2}{*}{ Gender } & Female & 33 & 32 \\
\hline & Male & 19 & 9 \\
\hline \multirow{6}{*}{ Age } & $<20$ & 4 & 4 \\
\hline & $20-24$ & 13 & 22 \\
\hline & $25-34$ & 21 & 8 \\
\hline & $35-49$ & 6 & 4 \\
\hline & 50-59 & 7 & 3 \\
\hline & $>60$ & 1 & 0 \\
\hline \multirow{3}{*}{ Education role } & Teacher & 20 & 4 \\
\hline & Student & 16 & 25 \\
\hline & Not in education & 16 & 12 \\
\hline \multirow{2}{*}{ Education level } & High Education & 39 & 37 \\
\hline & Non High Education & 13 & 4 \\
\hline
\end{tabular}

Table 1. Summary statistics for the demographic breakdown of the participants.

conducted, lasting on average for 35 minutes. The users were expected to stay standing throughout the course of the experiment. Precautionary measures were taken to ensure the safety of participants. The experiment procedure and tasks were known to the participants before conducting the experiment. Participants were advised to express their satisfaction or dissatisfaction freely and to explain their experience in answering the questions. Users were invited to use the application's web version after experiencing the VR application and complete the evaluation survey comprising the two questionnaires, described above.

The participants in the control group (Web Only) were invited to try the web application without having to experience the VR version. They were invited by mail containing a link to the application and a link to access the survey containing only the first questionnaire. The invitations were sent to more than 80 potential participants, matching the number and demographic composition of the first group, with 41 positive responses. Demographic data for both groups are summarised in Table 1.

\subsection{Analysis and Results}

This section describes the observations and analysis of the findings from Section 4.2. Analysis of the results from the first questionnaire were conducted in the following steps: validity and reliability of the instrument is assessed using the Cronbach-alpha test, exploratory data analysis influenced the choice of the Chi-squared method to determine the statistical difference between results in two groups, and, finally, in the comparison of the overall results. The questionnaire structure and overview of statistical measures, the Mean $(\mu)$ and STD $(\sigma)$, for two groups are presented in Table 2.

The mean values presented in Table 2 for Performance Expectancy items are in the range of 5.19 and 6.10 for the Web after VR group and in the range of 4.44 and 5.78 for the Web Only group. Mean values for Effort Expectancy items are in the range of 5.44 and 5.92 for the Web after VR group and in the range of 4.98 and 5.58 for the Web Only group. Notable higher mean values indicate a non-symmetric distribution of answers.

Items addressing Performance Expectancy were designed to accommodate the specific context of the applications for preservation of Intangible Cultural Heritage. Since this example is not similar to any previous research, we tested the internal consistency of responses, calculating the Cronbach Alpha values for the basic constructs in 
1:14 Selmanović and Rizvic, et al.

\begin{tabular}{|c|c|c|c|c|}
\hline \multirow[b]{2}{*}{ Item } & \multicolumn{2}{|l|}{$\mu$} & \multicolumn{2}{|l|}{$\sigma$} \\
\hline & Web after VR & Web Only & Web after VR & Web Only \\
\hline $\begin{array}{l}\text { PE.1 - Using application I felt playful and imagi- } \\
\text { native }\end{array}$ & 5.88 & 5.51 & 1.52 & 1.27 \\
\hline PE.2 - I had a realistic feeling of a height & 5.52 & 4.46 & 1.63 & 1.57 \\
\hline $\begin{array}{l}\text { PE. } 3 \text { - I had a real experience of Mostar Old Bridge } \\
\text { diving }\end{array}$ & 5.19 & 4.59 & 1.66 & 1.34 \\
\hline $\begin{array}{l}\text { PE. } 4 \text { - My movements through the app were spon- } \\
\text { taneous }\end{array}$ & 5.60 & 5.29 & 1.38 & 1.44 \\
\hline $\begin{array}{l}\text { PE. } 5 \text { - I had a real experience of being at the Old } \\
\text { Bridge }\end{array}$ & 5.50 & 5.56 & 1.75 & 1.40 \\
\hline PE.6 - I listened to the stories with full attention & 5.05 & 5.29 & 1.91 & 1.54 \\
\hline $\begin{array}{l}\text { PE. } 7 \text { - I have learned interesting information about } \\
\text { the Old Bridge }\end{array}$ & 5.96 & 5.73 & 1.30 & 1.25 \\
\hline $\begin{array}{l}\text { PE. } 8 \text { - Application provided me with a unique } \\
\text { experience }\end{array}$ & 6.10 & 5.39 & 1.39 & 1.30 \\
\hline EE.1 - Using the application was easy & 5.83 & 5.07 & 1.42 & 1.52 \\
\hline $\begin{array}{l}\text { EE. } 2 \text { - I did not have problems to navigate through } \\
\text { the stories. }\end{array}$ & 5.87 & 4.76 & 1.48 & 1.89 \\
\hline EE.3 - Interaction is clear and understandable & 5.79 & 5.41 & 1.47 & 1.26 \\
\hline $\begin{array}{l}\text { EE.4 - Interaction with the application is not too } \\
\text { demanding }\end{array}$ & 5.92 & 5.24 & 1.37 & 1.64 \\
\hline $\begin{array}{l}\text { EE.5 - I did not need any assistance in using the } \\
\text { application }\end{array}$ & 5.44 & 5.44 & 1.76 & 1.61 \\
\hline
\end{tabular}

Table 2. Questions are split by evaluation construct, with PE.x directed towards Performance Expectancy and EE.x addressing Effort Expectancy. Responses were delivered on a 7-point Likert scale, with 1-strongly disagree and 7-strongly agree. Respective summary statistics measures are shown: Mean and Standard deviation, for both user groups: "Web after VR" and "Web Only"

our study. Cronbach Alpha for the Performance Expectancy is 0.90 and for the Effort Expectancy is 0.82 , which is considered excellent and very good respectively.

In order to validate our hypotheses the next step was to examine if there is an existing relationship between the two groups. The hypothetical dependence among the constructs was examined by calculating the following indices: chi-square $\left(\chi^{2}\right)$ value and degree of freedom $(d f)$. Exploratory visualisation of the results motivated this approach, which is in compliance with the small number of variables in our study, and with a sample size for each group slightly above 40 . The results in Table 3 indicate that there is a correlation between two groups for the Effort Expectancy, but there is a significant difference between results for the Performance Expectancy. We can conclude that experience with the VR did not affect Effort Expectancy for the web application, but the same experience made a significant influence on the Performance Expectancy construct.

A further step in comparing the results for the two groups would be to evaluate the difference between the two groups, using frequency measures as Top Agree and Percent Agree [34]. Top Agree represents the proportion of participants who reported a score of 7 on the 7-point scale, Percent Agree refers to the proportion that recorded a score of 5,6 or 7. Measures are presented in Table 4. Comparing data for PE items is more relevant, since we need to evaluate the influence of the VR experience. It is notable that measures for the PE items is consistently higher. 


\begin{tabular}{lcccc}
\hline Construct & $\chi^{2}$ & $d f$ & $\frac{\chi^{2}}{d f}$ & p-value \\
\hline Performance Expectancy & 57.37 & 6 & 9.56 & $<.00001$ \\
Effort Expectancy & 11.97 & 6 & 1.99 & .0626 \\
\hline
\end{tabular}

Table 3. Chi-square value $\chi^{2}$, degree of freedom $d f$, normalised chi-square $\frac{\chi^{2}}{d f}, \mathrm{p}$-value (the result is not significant at $\mathrm{p}>$ $.05)$

\begin{tabular}{ccccc}
\hline & \multicolumn{2}{c}{ Top Agree } & \multicolumn{2}{c}{ Percent Agree } \\
Item & Web after VR & Web Only & Web after VR & Web Only \\
\hline PE.1 & $47 \%$ & $28 \%$ & $87 \%$ & $93 \%$ \\
PE.2 & $40 \%$ & $11 \%$ & $77 \%$ & $49 \%$ \\
PE.3 & $26 \%$ & $5 \%$ & $79 \%$ & $56 \%$ \\
PE.4 & $28 \%$ & $14 \%$ & $87 \%$ & $83 \%$ \\
PE.5 & $38 \%$ & $41 \%$ & $79 \%$ & $78 \%$ \\
PE.6 & $34 \%$ & $24 \%$ & $77 \%$ & $76 \%$ \\
PE.7 & $43 \%$ & $52 \%$ & $89 \%$ & $80 \%$ \\
PE.8 & $58 \%$ & $37 \%$ & $87 \%$ & $78 \%$ \\
\hline EE.1 & $44 \%$ & $22 \%$ & $87 \%$ & $40 \%$ \\
EE.2 & $48 \%$ & $27 \%$ & $87 \%$ & $38 \%$ \\
EE.3 & $44 \%$ & $24 \%$ & $79 \%$ & $42 \%$ \\
EE.4 & $46 \%$ & $29 \%$ & $88 \%$ & $42 \%$ \\
EE.5 & $37 \%$ & $32 \%$ & $77 \%$ & $44 \%$ \\
& & & &
\end{tabular}

Table 4. Performance Expectancy and Effort Expectancy comparison. Questions are split by the evaluation construct, with PE.x directed towards Performance Expectancy and EE.x addressing Effort Expectancy. Responses were delivered on a 7-point Likert scale, with 1-strongly disagree and 7-strongly agree.

The VR experience influenced transfer of immersion into using the web version of the same application and this is supported by the finding presented in Table 3. It is interesting to note that items with different correlation are PE.6 ("I listened to the stories with full attention") and PE.7 ("I have learned interesting information about the Old Bridge"). This is in compliance with other sources indicating the limitation of educational performance due to higher immersion [23].

A second questionnaire was designed for direct comparison of two technologies: VR and Web multimedia. We added two items linked to Behaviour Intention. Questions aligned to BI were only used on the Web After VR group:

BI1: I would rather use the Mostar diving VR application again.

BI2: I would recommend the Mostar diving VR application to a friend.

We tested internal consistency for the sub-scales calculating Cronbach Alpha values for the constructs, with a result higher than 0.85 , indicating very good consistency. The distribution of responses for each specific item in the second questionnaire is presented in Figure 9; shown with a stacked bar chart, produced with code from [31]. The significant difference in distribution of responses is obvious. Distribution of responses for the Effort 


\begin{tabular}{lc}
\hline Hypothesis & Assessment \\
\hline H1. VR experience has a positive effect on web application PE. & Supported \\
H2. VR experience has a negative effect on web application EE. & Not supported \\
H3. VR experience will have a significant positive influence on Behavioral & Supported \\
Intention to use the VR. & \\
\hline
\end{tabular}

Table 5. Summary of hypotheses testing and conclusion, supported by the results.

Expectancy is showing that almost half of the participants consider both technologies as equal, while the rest of the answers are divided. This is in compliance with the results from the literature [67] indicating that with experience, users develop a certain level of expertise and for such users EE is decreasing its relevance for the Behaviour Intention construct. Considering the distribution of answers for PE and BI, the prevalence of VR as a technology of choice for this purpose is obvious. This is shown in Table 5.

\section{VR or Web preference}

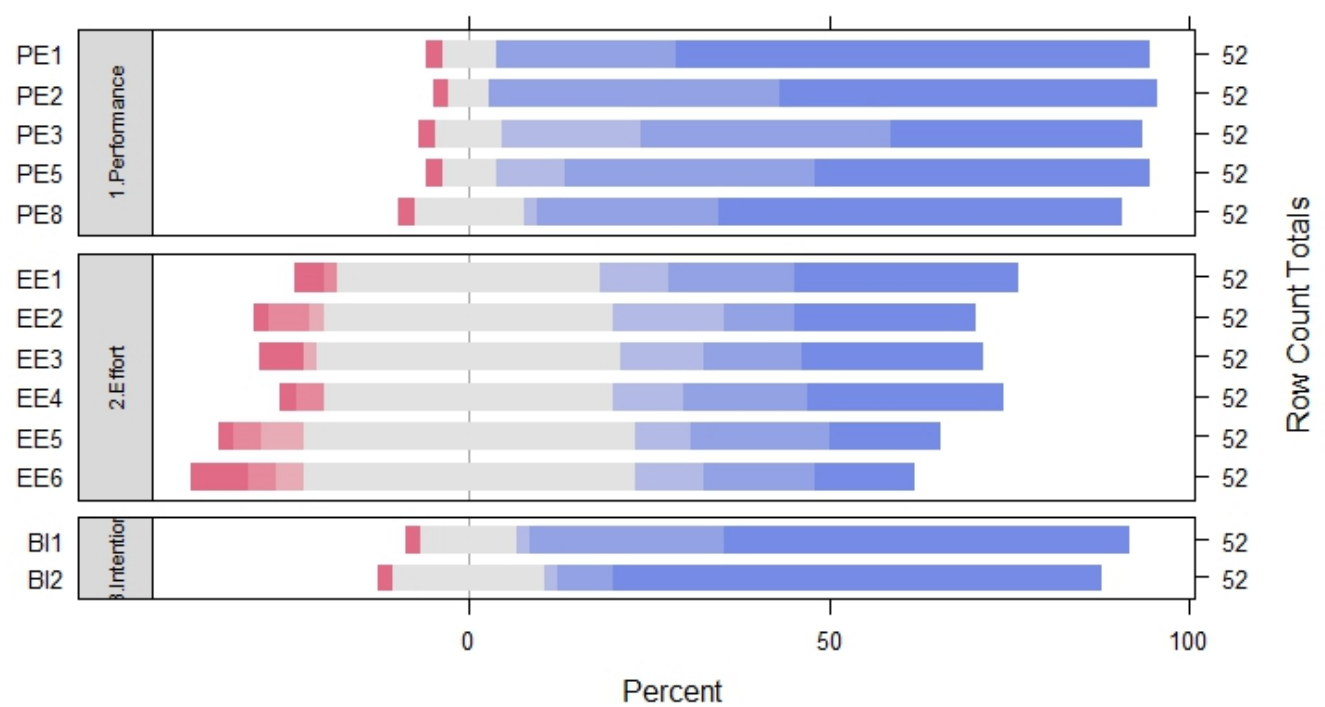

Web.strongly $\square$ Web $\square$ Web.slightly Equal $\square \quad$ VR.slightly $\square$ VR $\square$ VR.strongly

Fig. 9. Distribution of responses across each item comparing VR and Web applications. Constructs: Performance Expectancy (PE), Effort Expectancy (EE) and Behaviour Intention (BI)

\section{CONCLUSIONS}

We explored an approach in this work to compare a Web-based application versus a Virtual Reality application through the medium of the UTAUT to investigate the affect on usability and accessibility for the context of preserving Intangible Cultural Heritage. For this exploration, the Old Bridge diving tradition in Mostar was used 
as a case study. For this intangible heritage, applications of current VR and Web Multimedia technologies are used to enhance user immersion and enhance preservation and pedagogy. The interactive digital storytelling medium was used and special attention was paid when designing the interface for a $360^{\circ}$ environment by integrating mechanisms for local and at-a-distance interaction, where appropriate, into the simulation. Simulation was used as an important mechanism to preserve the heritage of Old Bridge Diving as it enabled the participant's empathy with a real-world diver via participation in the virtual world. This process was shown to create an intimate and personal connection to the tradition [56].

This work focused on extending this previous study to investigate the influence that prior exposure has with intangible heritage reconstructions and how this exposure influences technology adoption constructs in future exposure with similar non-VR content. A control group (Web Only) with no prior experience with the application developed were exposed to the web-based version presented in this manuscript. Another group (Web After VR) were also exposed to the web-based version, but after their exposure to the VR version of the same application. The evaluation results are indicating that the VR experience has a positive effect on web application adoption constructs around immersion and presence, irrelevant of the ease-of-use. We can even conclude that the path of influence is more likely from Performance Expectancy to Effort Expectancy and not in the opposite direction as modeled in TAM [17]. But such a conclusion, although reasonable, needs to be tested more rigorously in future studies.

For preservation, it's not only important to document heritage, but to transfer the intangible part that may include feelings, perception, drive, and motives. On the continuum of reality, from functional to photographic to physical reality, it is clear that many feelings and emotions testament to being there at the moment are afforded to the divers and wider heritage community. Traditional media does not excite the same level of participants' feelings that would indicate that this is somewhere low on the continuum of reality, whereas VR yields feelings of immersion and presence that are more closely aligned with that of physical reality and being there in the moment. It is also clear and the evaluation shown in Figure 9 supports that VR was the superior medium compared to more traditional methods across the technology adoption constructs. So whilst VR is the preferred medium to support intangible heritage applications moving forward, it is also shown that VR supports immersion and presence without negatively influencing ease-of-use in traditional forms of media that present intangible heritage.

\subsection{Limitations}

The technology considered $360^{\circ}$ field-of-view image presentation for the most part, correlating with the visual domain. However, the digital story experience could be enhanced by integrating $360^{\circ}$ acoustic signals into the VE. In addition, it is possible to separate the groups identified in the demographic capture rather than collapse. This makes it possible to gain some additional insight into the nature of preconditioning and the opinion of these different groups when considering this form of preservation through inductive analysis. The group that knew about the heritage, for example, rated story elements more favorably than the other group. Demographic analysis across factors such as Education can also be facilitated through this process. In the design of future preservation systems, insight into these elements is important.

\section{FUTURE WORK}

Future work will seek to synergise a framework for preserving intangible heritage so that recommendations are made to address the challenges of intangible heritage: validity, inclusivity, representativity, temporality, sensitivity and community. One avenue to explore this is via Structural Equation Modelling of the UTAUT framework responses provided for this context. This would empower a factor analysis for the elements for technology adoption in the context of intangible heritage. 
From the technical aspect, simulation could be improved so it includes physically-based elements. For example, a temporal site environment map could be captured and used. Some of the 3D models including trees and people could be animated. It would be interesting to explore which level of fidelity would be required to elicit the equivalent bio-metric and emotional response from the diver.

In addition, it was identified during the interviews that there are many diving styles that have been practised in Mostar over the years to supplement this tradition. The techniques may have adapted and evolved over time as diving heritage develops. Some of these dives may no longer be practiced. To further expand the preservation of the heritage, a motion capture database of these dives should be created. Considering immersion and presence, this database should have the provision to explore and experience these types of dives from a diver's reference frame.

\section{ACKNOWLEDGEMENTS}

We would like to thank all the participants who volunteered in the user study. This research was partially supported by the NVIDIA Corporation with the donation of the Titan Xp GPU.

\section{REFERENCES}

[1] 3D CoForm 2009. 3D CoForm Website. http://www.culturalinformatics.org.uk/. Accessed: 2018-06-14.

[2] Ward R. Ahmed E. 2016. Analysis of factors influencing acceptance of personal, academic and professional development e-portfolios. Campus-Wide Information Systems 63 (2016), 152-161.

[3] Irene Aicardi, Filiberto Chiabrando, Andrea Maria Lingua, and Francesca Noardo. 2018. Recent trends in cultural heritage 3D survey: The photogrammetric computer vision approach. Journal of Cultural Heritage 32 (2018), 257-266.

[4] Eike Anderson, Leigh McLoughlin, Fotis Liarokapis, Christopher Peters, Panagiotis Petridis, and Sara de Freitas. 2010. Developing serious games for cultural heritage: A state-of-the-art Review. Virtual Reality 14 (12 2010), 255-275.

[5] Ferran Argelaguet and Carlos Andujar. 2013. A survey of 3D object selection techniques for virtual environments. Computers \& Graphics 37, 3 (2013), 121-136.

[6] L. Argyriou, D. Economou, and V. Bouki. 2017. 360-degree interactive video application for Cultural Heritage Education. In 3rd Annual International Conference of the Immersive Learning Research Network (2017). 297-304.

[7] D. Arnold and G. Geser. 2007. Research Agenda for the Applications of ICT to Cultural Heritage. (2007).

[8] Ruth Aylett. 2000. Emergent narrative, social immersion and "storification". In Proceedings of the 1st International Workshop on Narrative and Interactive Learning Environments. 35-44.

[9] Bouchard Stéphane Bertrand Manon. 2008. Applying the technology acceptance model to VR with people who are favorable to its use. fournal of Cyber Therapy \& Rehabilitation 1, 2 (2008), 200-210.

[10] Doug A Bowman and Chadwick A Wingrave. 2001. Design and evaluation of menu systems for immersive virtual environments. In Virtual Reality, 2001. Proceedings. IEEE. IEEE, 149-156.

[11] Plewe Brandon. 2002. The Nature of Uncertainty in Historical Geographic Information. Transactions in GIS 6, 4 (2002), 431-456.

[12] Erik Champion. 2015. Entertaining The Similarities And Distinctions Between Serious Games and Virtual Heritage Projects. Entertainment Computing 14 (11 2015)

[13] Dimitrios Christopoulos, Pavlos Mavridis, Anthousis Andreadis, and John N. Karigiannis. 2013. Digital Storytelling within Virtual Environments: "The Battle of Thermopylae". Springer Berlin Heidelberg, Berlin, Heidelberg, 29-48. https://doi.org/10.1007/978-3-64237042- $7_{2}$

[14] Karin Coninx, Frank Van Reeth, and Eddy Flerackers. 1997. A hybrid 2D/3D user interface for immersive object modeling. In Computer Graphics International, 1997. Proceedings. IEEE, 47-55.

[15] Raimund Dachselt and Anett Hübner. 2007. Three-dimensional menus: A survey and taxonomy. Computers \& Graphics 31, 1 (2007), 53-65.

[16] Fred D Davis. 1986. A technology acceptance model for empirically testing new end-user information systems : theory and results /. Ph.D. Dissertation. MIT Sloan School of Management.

[17] Fred D. Davis. 1989. Perceived Usefulness, Perceived Ease of Use, and User Acceptance of Information Technology. MIS Q. 13, 3 (Sept. 1989), 319-340. https://doi.org/10.2307/249008

[18] Fred D. Davis, Richard P. Bagozzi, and Paul R. Warshaw. 1989. User Acceptance of Computer Technology: A Comparison of Two Theoretical Models. Manage. Sci. 35, 8 (Aug. 1989), 982-1003. https://doi.org/10.1287/mnsc.35.8.982

[19] H. Denard and S. Hermon. 2009. London Charter. http://www.londoncharter.org/history.html. Accessed: 2018-06-14. 
[20] Yuanfa Dong, Mark Webb, Carlo Harvey, Kurt Debattista, and Alan Chalmers. 2017. Multisensory Virtual Experience of Tanning in Medieval Coventry. In Eurographics Workshop on Graphics and Cultural Heritage, Tobias Schreck, Tim Weyrich, Robert Sablatnig, and Benjamin Stular (Eds.). The Eurographics Association. https://doi.org/10.2312/gch.20171297

[21] Declan Dowling, Colm O Fearghail, Aljoscha Smolic, and Sebastian Knorr. 2018. Faoladh : A Case Study in Cinematic VR Storytelling and Production. In International Conference for Interactive Digital Storytelling.

[22] Ahmed Elmezeny, Nina Edenhofer, and Jeffrey Wimmer. 2018. Immersive Storytelling in 360-Degree Videos: An Analysis of Interplay Between Narrative and Technical Immersion. Journal of Virtual Worlds Research 11 (04 2018).

[23] Pandey V. Fagan M, Kilmon C. 2008. Educational tool or expensive toy? Evaluating VR evaluation and its relevance for virtual heritage. Routledge, 242-260.

[24] Pandey V. Fagan M, Kilmon C. 2012. Exploring the adoption of a virtual reality simulation: The role of perceived ease of use, perceived usefulness and personal innovativeness. Campus-Wide Information Systems 29, 2 (2012), 117-127.

[25] Dominique Gerber and Dominique Bechmann. 2005. The spin menu: A menu system for virtual environments. In Virtual Reality, 2005. Proceedings. VR 2005. IEEE. IEEE, 271-272.

[26] A. Grande and V. Bendicho. 2011. The principles of the sevilla charter. http://smartheritage.com/seville-principles/seville-charter-project. Accessed: 2018-06-14.

[27] Sarajevo Graphics Group. 2018. Mostar Cliff Diving VR - webGL version. http://h.etf.unsa.ba/mostar-jumps/WebGLEng/. Accessed: 2019-03-27.

[28] Sarajevo Graphics Group. 2018. Sarajevo Charter. http://h.etf.unsa.ba/sarajevocharter/. Accessed: 2019-03-27.

[29] Pierre Grussenmeyer, Tania Landes, Thomas Voegtle, and Konrad Ringle. 2008. Comparison methods of terrestrial laser scanning, photogrammetry and tacheometry data for recording of cultural heritage buildings. International Archives of Photogrammetry, Remote Sensing and Spatial Information Sciences 37, B5 (2008), 213-218.

[30] Jassim Happa, Thomas Bashford-Rogers, Alexander Wilkie, Alessandro Artusi, Kurt Debattista, and Alan Chalmers. 2012. Cultural Heritage Predictive Rendering. Computer Graphics Forum 31, 6 (2012), 1823-1836.

[31] Richard Heiberger and Naomi Robbins. 2014. Design of Diverging Stacked Bar Charts for Likert Scales and Other Applications. Fournal of Statistical Software 57, 5 (April 2014), 1-32.

[32] Vedad Hulusic, Carlo Harvey, Kurt Debattista, Nicolas Tsingos, Steve Walker, David Howard, and Alan Chalmers. 2012. Acoustic Rendering and Auditory-Visual Cross-Modal Perception and Interaction. Computer Graphics Forum 31, 1 (2012), 102-131. https: //doi.org/10.1111/j.1467-8659.2011.02086.x

[33] I. Ivkovic, N. Klisura, and S. Sljivo. 2018. Bridges of Sarajevo. In Central European Seminar on Computer Graphics (2018). 107-114.

[34] J.R: Lewis J. Sauro. 2016. Quantifying the user experience: Practical statistics for user research. Morgan Kaufmann.

[35] Richard H Jacoby and Stephen R Ellis. 1992. Using virtual menus in a virtual environment. In Visual Data Interpretation, Vol. 1668. International Society for Optics and Photonics, 39-49.

[36] Jonathan Lazar, Jinjuan Heidi Feng, and Harry Hochheiser. 2010. Research Methods in Human-Computer Interaction. Wiley Publishing.

[37] Patrick Lemoine, Frederic Vexo, and Daniel Thalmann. 2003. Interaction techniques: 3d menus-based paradigm. AVIR 2003 VRLABCONF-2007-030 (2003).

[38] Sandy Louchart and Ruth Aylett. 2003. Solving the Narrative Paradox in VEs - Lessons from RPGs. In Intelligent Virtual Agents, Thomas Rist, Ruth S. Aylett, Daniel Ballin, and Jeff Rickel (Eds.). Springer Berlin Heidelberg, Berlin, Heidelberg, 244-248.

[39] Choi D. Manis KT. 2018. The virtual reality hardware acceptance model (VR-HAM): Extending and individuating the technology acceptance model (TAM) for virtual reality hardware. Journal of Business Research (2018).

[40] Bonn Maria, Kendall Lori, and McDonough Jerome. 2016. Preserving intangible heritage: Defining a research agenda. Proceedings of the Association for Information Science and Technology 53, 1 (2016), 1-5.

[41] John McCarthy. 2014. Multi-image photogrammetry as a practical tool for cultural heritage survey and community engagement. fournal of Archaeological Science 43 (2014), 175-185.

[42] Daniel Mendes, Daniel Medeiros, Maurício Sousa, Eduardo Cordeiro, Alfredo Ferreira, and Joaquim A Jorge. 2017. Design and evaluation of a novel out-of-reach selection technique for VR using iterative refinement. Computers \& Graphics 67 (2017), 95-102.

[43] Helen C Miles, Andrew T Wilson, Frédéric Labrosse, Bernard Tiddeman, Seren Griffiths, Ben Edwards, Panagiotis D Ritsos, Joseph W Mearman, Katharina Möller, Raimund Karl, et al. 2016. Alternative representations of 3D-reconstructed heritage data. fournal on Computing and Cultural Heritage ( (JOCCH) 9, 1 (2016), 4.

[44] Hermitage Museum and Videofabrika. 2017. The Hermitage VR Experience. http://www.inavateonthenet.net/case-studies/article/ immersive-history-russia-s-hermitage-museum-embraces-vr. Accessed: 2018-06-14.

[45] F. Nicolucci and S. Hermon. 2004. A Fuzzy Logic Approach to Reliability in Archaeological Virtual Reconstruction. In Beyond the Artifact (1968), F. Nicolucci and S. Hermon (Eds.). 28-35.

[46] Cecilia Pisa, Fabiana Zeppa, and Gabriele Fangi. 2011. Spherical Photogrammetry for Cultural Heritage\&Mdash;San Galgano Abbey and the Roman Theater, Sabratha. J. Comput. Cult. Herit. 4, 3, Article 9 (Dec. 2011), 15 pages. https://doi.org/10.1145/2069276.2069278 
[47] Athena Plus. 2017. Digital cultural heritage and tourism recommendations for cultural institutions. http://www.athenaplus.eu/ index.php?en/220/digital-cultural-heritage-and-tourism-recommendations-for-cultural-institutions. Accessed: 2018-06-14.

[48] Fabio Remondino, Stefano Girardi, Alessandro Rizzi, and Lorenzo Gonzo. 2009. 3D Modeling of Complex and Detailed Cultural Heritage Using Multi-resolution Data. f. Comput. Cult. Herit. 2, 1, Article 2 (July 2009), 20 pages. https://doi.org/10.1145/1551676.1551678

[49] Selma Rizvic, Dusanka Boskovic, Vensada Okanovic, and Sanda Sljivo. 2017. Kyrenia - Hyper Storytelling Pilot Application. In Eurographics Workshop on Graphics and Cultural Heritage, Tobias Schreck, Tim Weyrich, Robert Sablatnig, and Benjamin Stular (Eds.) The Eurographics Association.

[50] S. Rizvic, N. Djapo, F. Alispahic, B. Hadzihalilovic, F. F. Cengic, A. Imamovic, V. Okanovic, and D. Boskovic. 2017. Guidelines for interactive digital storytelling presentations of cultural heritage. In 2017 9th International Conference on Virtual Worlds and Games for Serious Applications (VS-Games)(VS-GAMES), Vol. 00. 253-259.

[51] Ruth and Aylett. 1999. Narrative in Virtual Environments-Towards Emergent Narrative.

[52] Paola Salomoni, Catia Prandi, Marco Roccetti, Lorenzo Casanova, Luca Marchetti, and Gustavo Marfia. 2017. Diegetic user interfaces for virtual environments with HMDs: a user experience study with oculus rift. Journal on Multimodal User Interfaces 11, 2 (2017), 173-184.

[53] Sarajevo Graphics Group. 2018. Mostar VR Gameplay. https://youtu.be/v-HoWOKOtMs.

[54] Sarajevo Graphics Group. 2018. VR simulation of Mostar bridge diving. https://tinyurl.com/sarajevographicsgroup.

[55] Henrik Schoenau-Fog. 2015. Adaptive Storyworlds: Utilizing the Space-Time Continuum in Interactive Digital Storytelling. In Interactive Storytelling, Henrik Schoenau-Fog, Luis Emilio Bruni, Sandy Louchart, and Sarune Baceviciute (Eds.). Springer, Germany, 58-65.

[56] Elmedin Selmanovic, Selma Rizvic, Carlo Harvey, Dusanka Boskovic, Vedad Hulusic, Malek Chahin, and Sanda Sljivo. 2018. VR Video Storytelling for Intangible Cultural Heritage Preservation. In Eurographics Workshop on Graphics and Cultural Heritage, Robert Sablatnig and Michael Wimmer (Eds.). The Eurographics Association. https://doi.org/10.2312/gch.20181341

[57] Rod Sims. 1997. Interactivity: A forgotten art? Computers in human behavior 13, 2 (1997), 157-180.

[58] Efstathios Stavrakis, Andreas Aristidou, Maria Savva, Stephania Loizidou Himona, and Yiorgos Chrysanthou. 2012. Digitization of Cypriot Folk Dances. In Progress in Cultural Heritage Preservation, Marinos Ioannides, Dieter Fritsch, Johanna Leissner, Rob Davies, Fabio Remondino, and Rossella Caffo (Eds.). Springer Berlin Heidelberg, Berlin, Heidelberg, 404-413.

[59] Jonathan Steuer. 1992. Defining virtual reality: Dimensions determining telepresence. fournal of communication 42, 4 (1992), 73-93.

[60] Stella Sylaiou, Katerina Mania, Ioannis Paliokas, Laia Pujol, Vassilis Killintzis, and Fotis Liarokapis. 2017. Exploring the educational impact of diverse technologies in online virtual museums. International fournal of Arts and Technology 10 (01 2017), 58.

[61] Bjoern Flindt Temte and Henrik Schoenau-Fog. 2012. Coffee Tables and Cryo Chambers: A Comparison of User Experience and Diegetic Time between Traditional and Virtual Environment-Based Roleplaying Game Scenarios. In Interactive Storytelling, David Oyarzun, Federico Peinado, R. Michael Young, Ane Elizalde, and Gonzalo Méndez (Eds.). Springer Berlin Heidelberg, Berlin, Heidelberg, 102-113.

[62] Ashima Thomas, Abhi Kumar, Race Krehel, Kay Vasey, Eng Tat Khoo, Tim Marsh, and Benjamin Li. 2018. Oceans we make: immersive VR storytelling. In SIGGRAPH Asia 2018 Virtual \& Augmented Reality. 1-2.

[63] UNESCO 2019. UNESCO Intangible Cultural Heritage. https://ich.unesco.org/en/what-is-intangible-heritage-00003. Accessed: 2019-09-01.

[64] ICH UNESCO. 2003. Text of the Convention for the Safeguarding of the Intangible Cultural Heritage.

[65] Marilena Vecco. 2010. A definition of cultural heritage: From the tangible to the intangible. Fournal of Cultural Heritage 11, 3 (2010), 321-324.

[66] Viswanath Venkatesh and Fred D. Davis. 1996. A Model of the Antecedents of Perceived Ease of Use: Development and Test*. Decision Sciences 27, 3 (1996), 451-481. https://doi.org/10.1111/j.1540-5915.1996.tb00860.x arXiv:https://onlinelibrary.wiley.com/doi/pdf/10.1111/j.1540-5915.1996.tb00860.x

[67] Viswanath Venkatesh, Michael G. Morris, Gordon B. Davis, and Fred D. Davis. 2003. User Acceptance of Information Technology: Toward a Unified View. MIS Quarterly 27 (2003), 425-478.

[68] Virtual Reality Toolkit 2018. VRTK - Virtual Reality Toolkit. https://vrtoolkit.readme.io. Accessed: 2018-06-14.

[69] Naci Yastikli. 2007. Documentation of cultural heritage using digital photogrammetry and laser scanning. fournal of Cultural Heritage 8 , 4 (2007), 423-427.

[70] Haci Murat Yilmaz, Murat Yakar, Saadet Armagan Gulec, and O Nuri Dulgerler. 2007. Importance of digital close-range photogrammetry in documentation of cultural heritage. Journal of Cultural Heritage 8, 4 (2007), 428-433. 